\title{
In the land of the monolingual
}

Translating a basic finding into a new therapy requires us to speak many languages-scientific, clinical, legal and financial. Yet most of us are hopelessly 'monolingual', a limitation that substantially slows translational research. Steps have been taken to address this problem, but a lot remains to be done.

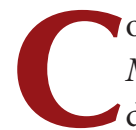
ongratulations! You've just published a paper in Nature Medicine identifying a new target to treat your favorite disease and are eager to take this finding to the clinic. Excellent, but where do you start? Are you familiar with all the steps you need to take from your discovery to a clinical trial?

Many of us would readily claim to be translational researchers, but, in reality, very few of us know how to navigate the translational pathway-we are very comfortable speaking the language of the lab but have a quite limited understanding of the plethora of legal (how to secure a patent), financial (how to raise money to develop our intellectual property), regulatory (how to interact with a regulatory agency) and clinical (how to recruit subjects for a clinical trial) issues that must be considered before we get anywhere near a new therapy.

Fortunately, various organizations in the US, including the National Institutes of Health (NIH), have started to tackle this problem by helping us build teams with all of the necessary skills to improve our chances of translational success. As we highlighted last year (Nat. Med. 14, 697; 2008) and in a recent Commentary (Nat. Med. 15, 502-507; 2009), the NIH grants five-year-long Clinical and Translational Science Awards (CTSAs) to universities and medical centers ( 46 so far, with a goal of 60 by 2012). The CTSAs hope to foster translational success by bringing together basic scientists, clinicians and other experts with knowledge crucial to walking the translational road. These human resources come together either physically (by creating new research labs and consortia) or virtually (through social networks and by posting resources on the CTSA website). CTSAs range from $\$ 20$ million to over $\$ 100$ million per institution, with over half a billion dollars awarded so far.

Resources, however, are not a panacea. A team leader in a translational venture should understand the entire process and be familiar with all of the obstacles that can be found along the way-a polyglot, instead of a monolingual. As noted in a Commentary in this issue (Nat. Med. 15, 1006-1009; 2009), training such a new type of professional individual is a priority. In the US, this need has led to the creation of a series of training programs, such as the Master in Translational Research of the University of Pennsylvania's Institute for Translational Medicine and Therapeutics (ITMAT), a CTSA consortium member. In Europe, the Wellcome Trust cosponsors Translational Medicine and Therapeutics PhD Programs at four sites in the UK, and Italy's Eureka Institute, alluded to in the above-mentioned Commentary, seeks to train future leaders in translational research by familiarizing them with every step of the translational pathway.

Although these training programs are geared toward junior scientists, there are also options for more senior researchers. For example, the ITMAT and other members of the CTSA consortium hold seminar series, symposia and workshops to educate researchers in all aspects of the translational process. However, what is still needed is a fully integrated, web-based information series, such as an online tutorial, hosted perhaps on an NIH-sponsored website, which can disseminate more broadly the key insights that researchers need to successfully cross the translational pathway.

A final example worth highlighting is the MaRS Centre in Canada, a local incubator that acts in conjunction with the University of Toronto to pair scientists with technology and entrepreneurs. A key feature of MaRS is a one-on-one mentoring program to assist scientists looking to make a commercial leap. Although the focus of the Centre is more on business than on research, the MaRS approach could be adapted to the CTSA model, providing senior scientists with advisors that could help their translational efforts. These advisors could be other scientists who have successfully made the translational jump or dedicated professionals (perhaps graduates from the training programs cited above) hired by universities or even the NIH.

All of the above initiatives are relatively new, reach a small number of scientists and are still experimental- the best ways to facilitate translational research have yet to be discovered. However, their sheer existence offers hope that translational research will become smoother, speeding up the rate at which drugs and medical devices make it to the clinic. At the same time, it is clear that we need to do a lot more on a global scale to empower scientists with the education necessary to make a real translational impact. If each of us tries to become at least 'bilingual', we might feel a little less overwhelmed by the Tower of Babel that translational research has become. 